\title{
Perception and Conception: Temporal Lobe Activity during Complex Discriminations of Familiar and Novel Faces and Objects
}

\author{
Morgan D. Barense ${ }^{1,2}$, Richard N. A. Henson ${ }^{3}$, and Kim S. Graham ${ }^{4}$
}

\begin{abstract}
Recent studies indicate that medial-temporal lobe (MTL) damage, either from focal lesions or neurodegenerative disease (e.g., semantic dementia), impairs perception as well as long-term declarative memory. Notably, however, these two patient groups show different performance for meaningful versus unfamiliar stimuli. In amnesics with nonprogressive MTL lesions, the use of meaningful stimuli, compared with unfamiliar items, boosted discrimination performance. In semantic dementia, a condition characterized by progressive deterioration of conceptual knowledge in the context of anterolateral temporal lobe damage, performance for meaningful stimuli was equivalent to that for unfamiliar items. To further investigate these findings, we scanned healthy volunteers while they performed odd-one-out discriminations involving familiar (i.e., meaningful/famous) and unfamiliar (i.e., novel) objects and faces and a baseline task of size oddity. Outside the scanner, volunteers' recognition memory was assessed.
\end{abstract}

\section{INTRODUCTION}

Structures in the medial-temporal lobe (MTL), including the hippocampus and connected areas (entorhinal, perirhinal, and parahippocampal cortex), have been proposed to constitute a system of structures responsible for long-term declarative memory, our conscious memory for facts and events (e.g., Eichenbaum \& Cohen, 2001; Squire \& Zola-Morgan, 1991). A central tenet of this view is that the primary function of the MTL is in memory and not other aspects of cognition, such as perception (Squire, Stark, \& Clark, 2004). Recent reports, however, challenge this view, suggesting instead that the MTL is not only important for memory but also essential for certain forms of perception (e.g., Lee \& Rudebeck, 2010; Barense, Gaffan, \& Graham, 2007; Lee, Buckley, et al., 2005; Bussey, Saksida, \& Murray, 2003; Buckley, Booth, Rolls, \& Gaffan, 2001). These findings led to an alternative characterization of MTL structures, which holds that apparently distinct mnemonic and perceptual functions may arise from common representations and computational mechanisms (Cowell, Bussey, \&

\footnotetext{
${ }^{1}$ University of Toronto, ${ }^{2}$ Rotman Research Institute, Toronto, Canada, ${ }^{3}$ MRC Cognition and Brain Sciences Unit, ${ }^{4}$ Cardiff University
}

We found above baseline activity in the perirhinal cortex and hippocampus for all object and face discriminations and above baseline activity in the temporal pole for all face discriminations. The comparison of meaningful, relative to novel, faces and objects, revealed increased activity in the perirhinal cortex and hippocampus. In the temporal pole, we also found activity related to meaningfulness for faces but not for objects. Importantly, these meaningfulness effects were evident even for discriminations that were not subsequently well remembered, suggesting that the difference between meaningful and novel stimuli reflects perceptual or conceptual processes rather than solely incidental encoding into long-term memory. The results provide further evidence that the MTL is recruited during complex perceptual discrimination and additionally suggest that these structures are recruited in semantic processing of objects and faces.
Saksida, 2010; Graham, Barense, \& Lee, 2010; Bussey \& Saksida, 2007; Murray, Bussey, \& Saksida, 2007). More specifically, recent lesion studies in both humans and monkeys suggest that perirhinal cortex is necessary for processing complex conjunctions of features comprising objects and faces during both memory and perceptual tasks (Lee \& Rudebeck, 2010; Barense et al., 2005, 2007; Taylor, Henson, \& Graham, 2007; Lee, Bussey, et al., 2005; Bussey, Saksida, $\&$ Murray, 2002). Functional neuroimaging of healthy participants has provided some support for these patient data (Barense, Henson, Lee, \& Graham, 2010; O’Neil, Cate, \& Kohler, 2009; Lee, Scahill, \& Graham, 2008; Devlin \& Price, 2007). For example, Barense, Henson, et al. (2010) found increased perirhinal activity for visual discriminations involving unfamiliar faces and unfamiliar objects (greebles) when the discrimination placed a higher demand on processing conjunctions of features (i.e., when the stimuli were shown from different, compared with same, viewpoints). A large body of research, however, indicates that MTL activity observed during incidental tasks can predict successful memory on subsequent memory tests (e.g., Diana, Yonelinas, \& Ranganath, 2007; Kensinger \& Schacter, 2006; Uncapher \& Rugg, 2005; Kirwan \& Stark, 2004; Ranganath et al., 2004; Davachi \& Wagner, 2002). Thus, one potential interpretation 
of some of the fMRI findings described above is that they reflect incidental encoding into long-term memory rather than perceptual processing per se.

It has also been proposed that the perirhinal cortex may be important for semantic memory (Murray \& Bussey, 1999). Given its widespread and polymodal afferents (Suzuki \& Amaral, 1994) and position at the boundary between putative mnemonic and perceptual regions, the perirhinal cortex is well placed to act as an interface between perceptual processing and conceptual knowledge. In support of this idea, some researchers have found evidence that the perirhinal cortex integrates cross-modal feature information into higher-level semantic memories of meaningful objects (Holdstock, Hocking, Notley, Devlin, \& Price, 2009; Taylor, Stamatakis, \& Tyler, 2009) and that it plays a critical role in conceptual implicit memory (Wang, Lazzara, Ranganath, Knight, \& Yonelinas, 2010). Studies with semantic dementia (SD) - a neurodegenerative condition characterized by a gradual, cross-modal deterioration of semantic knowledge and striking atrophy to anterior-inferior temporal regions, including the perirhinal cortex (Lee \& Rudebeck, 2010; Lee et al., 2006; Davies, Graham, Xuereb, Williams, \& Hodges, 2004; Davies, Xuereb, \& Hodges, 2002; Chan et al., 2001; Galton et al., 2001) — provide support for this idea. These studies have highlighted a role for the anterior temporal lobe as an amodal semantic hub thought to support the interactive activation of surface representations across different modalities (e.g., shape, action, and color; Binney, Embleton, Jefferies, Parker, \& Ralph, 2010; Visser, Jefferies, \& Lambon Ralph, 2010; Patterson, Nestor, \& Rogers, 2007; Rogers et al., 2004; McClelland \& Rogers, 2003).

A recent study highlighted the importance of semantic knowledge on visual discrimination tasks with no overt semantic component in individuals with temporal lobe damage (Barense, Rogers, Bussey, Saksida, \& Graham, 2010). Across a series of odd-one-out perceptual tasks, stimulus meaningfulness differentially influenced performance in patients with SD compared with amnesics with focal MTL lesions that included the hippocampus and perirhinal cortex bilaterally. Although the amnesic patients with focal MTL lesions were impaired on discriminations of both novel and meaningful stimuli, in both tasks, their deficit was attenuated by the use of meaningful stimuli. In contrast to the MTL amnesics, the SD patients showed no facilitation from the use of semantically familiar/meaningful stimuli. Thus, although they were impaired on both familiar and novel discriminations, the MTL cases were able to engage semantic support that was presumably not available to the SD patients (see also MacKay \& James, 2009; MacKay, James, \& Hadley, 2008; Moses, Ostreicher, Rosenbaum, \& Ryan, 2008). Unfortunately, given the diffuse nature of the lesions in both these patient groups, it was difficult to determine the neuroanatomical correlates of these effects. Thus, to provide greater neuroanatomical resolution, the present study used fMRI of healthy individuals while they performed nearly identical visual discriminations of objects and faces with varying levels of stimulus familiarity (note that by "familiarity," we refer to whether a representation of the stimulus existed before scanning). To investigate effects of incidental encoding, participants' recognition memory for the object and face stimuli was assessed outside the scanner and used to back-sort activity according to how well the item was encoded. We predicted that contrasts investigating stimulus meaningfulness (i.e., Familiar Faces-Unfamiliar Faces and Familiar Objects-Unfamiliar Objects) would elicit more activity in the temporal pole and perirhinal cortex-areas damaged by SD (Barense, Rogers, et al., 2010). Moreover, we predicted that this activity would, at least in part, reflect perceptual and conceptual processes additional to memory encoding processes and so would still be present even for stimuli that were not subsequently well remembered.

\section{METHODS \\ Participants}

Eighteen right-handed neurologically normal participants were scanned ( 12 women, mean age $=27.3$ years, standard deviation $=5.5$ ). After the nature of the study and its possible consequences had been explained, all participants gave informed written consent. This work received ethical approval from the Cambridgeshire Local Research Ethics Committee (LREC reference 05/Q0108/127).

\section{Image Acquisition}

The scanning was performed using a Siemens 3 T TIM Trio. Four sessions were acquired for every participant. Because of concerns about susceptibility-induced signal distortion and signal loss in the anterior MTLs, a dual-echo sequence (spin-echo and gradient-echo; Schwarzbauer, Mildner, Heinke, Brett, \& Deichmann, 2010) was used to acquire T2*-weighted volumes with BOLD contrast. Analysis of the spin-echo data (which is less prone to susceptibility artifacts) did not reveal any additional significant effects to the more standard gradient-echo data; consequently, only the latter are reported here. The slices were acquired in an interleaved sequence of 32 slices (16 spin-echo and 16 gradientecho) that followed the temporal lobes (see Figure 5 for coverage of the brain) and were tilted down by approximately $30^{\circ}$ to follow the axis of the hippocampus (slice thickness $=3 \mathrm{~mm}$, gap $=1 \mathrm{~mm}$, matrix size $=64 \times 64$, in-plane resolution $=3.5 \times 3.5 \mathrm{~mm}, \mathrm{TR}=2000 \mathrm{msec}$ ). The first three EPI sessions were $630 \mathrm{sec}$ in duration (5 dummy scans at the start to allow the MRI signal to reach equilibrium and 310 subsequent data scans), and the fourth EPI session was $542 \mathrm{sec}$ in duration (5 dummy scans and 266 subsequent data scans). A T1 structural scan was acquired for each participant using an MPRAGE sequence $(\mathrm{TR}=$ $2250 \mathrm{msec}$, TE $=2.99 \mathrm{msec}$, flip angle $=9^{\circ}$, field of view $=$ $256 \times 240 \times 160 \mathrm{~mm}$, matrix size $=256 \times 240 \times 160 \mathrm{~mm}$, spatial resolution $=1 \times 1 \times 1 \mathrm{~mm}$ ).

Visual stimuli were presented during scanning with a program written using E-Prime software (Psychology Software 
Tools, Inc., Pittsburgh, PA). The program was run on an IBM compatible desktop computer connected to an LCD projector (resolution $=1024 \times 768$ pixels) that projected onto a white screen situated behind the scanner bed. The screen could be seen with an angled mirror placed directly above the participant's eyes in the scanner. The responses for the experimental task were made using three specified buttons on a four-button response box held in the right hand. Response times and accuracy were automatically recorded by the computer.

\section{Experimental Paradigm}

Participants were administered a series of oddity discrimination tests, in which they were instructed to choose the unique stimulus from an array of three simultaneously presented items. They were told that of the three pictures presented per trial, two depicted the same stimulus, whereas the third picture was of a different stimulus. It is important to emphasize that for each oddity trial the three pictures were presented simultaneously, thus minimizing mnemonic demands of the task. Furthermore, to minimize mnemonic demands across trials, all stimuli were trial-unique. There were four stimulus types of interest, famous faces, familiar objects, unfamiliar faces, and novel objects (greebles), plus an additional control stimulus (black squares; Figure 1). For all five conditions, the three images (faces, objects, or squares) were presented on a white screen with one stimulus positioned above the remaining two. Each trial lasted $5.75 \mathrm{sec}$ (5.5-sec stimulus display time, 0.25 -sec intertrial interval), during which the participants were required to select the odd-one-out by pressing the corresponding button on the response box as quickly but as accurately as possible. The location of the odd stimulus in the array was counterbalanced across each condition. Each condition was presented in a miniblock of three trials each. The order of miniblocks was fixed for each participant and counterbalanced across participants. Two short practice sessions with feedback (one outside and one inside the scanner) were administered before the start of scanning. The first three EPI sessions consisted of 105 trials, and the fourth EPI session consisted of 90 trials.

Each of the five conditions contained 81 trials and was designed according to the following specific parameters:

\section{Face Oddity (Familiar and Unfamiliar Conditions)}

On each trial, three grayscale images of White human faces were presented on a white background (each individual face was overlaid on a black background of $170 \times 216$ pixels). Two of the images were of the same face taken from different viewpoints, whereas the third image was of a different face taken from another view. All faces were trial-unique. There were two different face oddity conditions: familiar

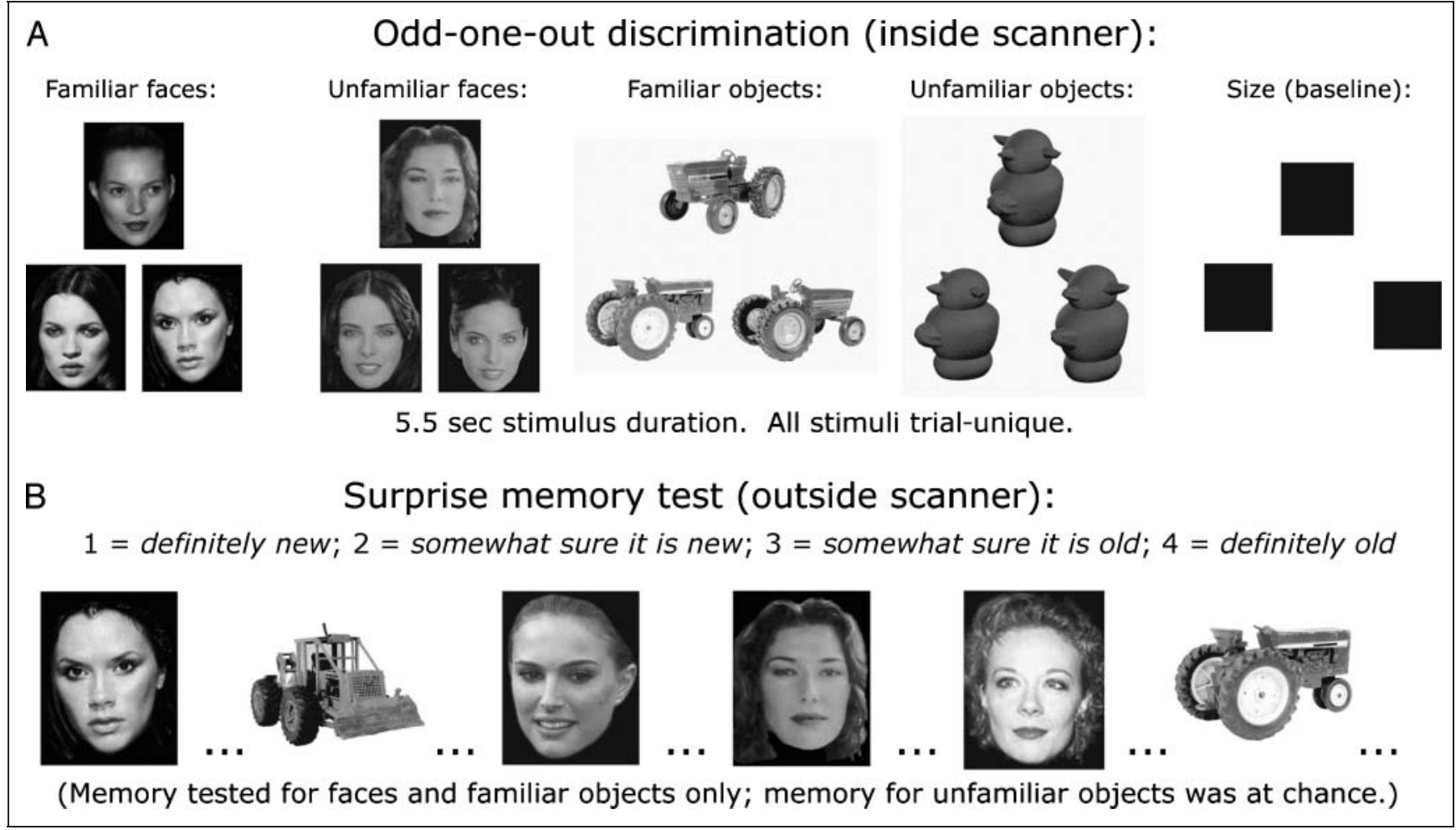

Figure 1. Example stimuli from the (A) oddity visual discrimination (five conditions) and (B) memory test portions of the experiment (figure depicts one example of a previously viewed stimulus [in scanner] and one example of a lure, for each of the three Familiar Face, Unfamiliar Face, Familiar Object conditions). For the oddity test, participants were told that of the three pictures presented per trial, two depicted the same stimulus, whereas the third picture was of a different stimulus. They were instructed to select the different stimulus. For each oddity trial, all stimuli were trial-unique and the three pictures were presented simultaneously, thus minimizing mnemonic demands of the task. 
and unfamiliar. The familiar condition comprised famous faces likely to be known to the participants, whereas the unfamiliar condition comprised nonfamous faces (equivalent to the Different View Face condition of Barense, Henson, et al., 2010). Each condition contained 40 trials containing female faces and 41 trials containing male faces.

\section{Object Oddity (Familiar and Unfamiliar Conditions)}

On each trial, three color images of objects were presented (each object occupied approximately $170 \times 216$ pixels). Two of the images were of the same object taken from two different viewpoints, whereas the third image was of a slightly different object taken from a third viewpoint. All objects were trial-unique. There were two different object oddity conditions: familiar and unfamiliar. The familiar condition comprised highly familiar everyday nonliving objects obtained from a database of 150,000 digital photo objects (Hemera Photo-Objects Volumes 1-3; Barense, Rogers, et al., 2010; Barense et al., 2007). For any given trial, the objects were from the same basic-level category, and extreme care was taken to ensure that the two objects shared a high number of overlapping features and the solution could not be obtained on the basis of a single, obvious feature. For the unfamiliar object condition, "greebles" were chosen because they represent a well-controlled set of novel, three-dimensional objects that are not perceived as faces except when the viewer is trained extensively (e.g., Gauthier \& Tarr, 1997). Such stimuli were also used successfully in our previous study (in the Different View Object condition of Barense, Henson, et al., 2010). None of our participants had any previous experience with greebles, and thus, the greebles served as object stimuli for which participants would have little or no pre-existing semantic representation. On any given trial, the greebles were always from the same family and the same gender and were of the same symmetry (i.e., asymmetrical versus symmetrical). Within those criteria, the greebles for each trial were selected to produce the maximum amount of possible feature overlap between the odd-one-out and the foils while matching difficulty to the other conditions. Difficulty was equated through a series of behavioral pilot experiments.

\section{Difficult Size Oddity (Baseline)}

On each trial, three black squares were presented. The length of each side was randomly varied from 67 to 247 pixels, and the size of each square was trial-unique. In each trial, two of the squares were the same size, whereas the third square was either larger or smaller. The difference between the lengths of the two different sides varied between 9 and 15 pixels. The positions of squares were jittered slightly so that the edges did not line up along vertical or horizontal planes. Through pilot experiments outside the scanner, the difficulty of this condition was designed to closely match that of the other oddity tasks.

\section{Surprise Memory Test (Postscanning)}

Approximately 30 min following the final scanning run, participants completed a surprise memory test outside the scanner for the Familiar Faces, Unfamiliar Faces, and Familiar Objects they had seen during the oddity test (pilot testing revealed that memory for unfamiliar objects and squares was at chance, so these conditions were omitted). During the memory test, a single stimulus was presented for $3.0 \mathrm{sec}$ and participants were asked to indicate via the keyboard their memory for the stimulus on a scale of $1-4$ $(1=$ definitely new, 2 = somewhat sure it is new, $3=$ somewhat sure it is old, $4=$ definitely old $)$. All 243 stimuli seen at study (i.e., 81 from each of the three tested conditions), intermixed with 180 new stimuli not seen during the oddity test ( 60 new stimuli from each of the three tested conditions), were presented in a pseudorandom order. For the trials involving previously seen stimuli, the presented stimulus had always been the odd-one-out during the oddity test. For the trials involving new familiar object stimuli, the foils were from the same basic-level category as the corresponding oddity trial (e.g., a tractor, a camera, etc.). These measures were used to analyze the oddity discrimination data according to subsequent memory (see Analysis 2 below).

\section{Image Preprocessing}

The fMRI data were preprocessed and analyzed using Statistical Parametric Mapping software (SPM5, www.fil.ion. ucl.ac.uk/spm/software/spm5/). Data preprocessing involved (1) realigning all images with respect to the first image of the first run via sinc interpolation and creating a mean image (motion correction), (2) undistorting the EPI data to correct for magnetic field distortions (Cusack \& Papadakis, 2002), and (3) normalizing each participant's structural scan to the Montreal Neurological Institute (MNI) ICBM152 T1 average brain template and applying the resulting normalization parameters to the EPI images. The normalized images were interpolated to $3 \times 3 \times 3 \mathrm{~mm}$ voxels and smoothed with an 8-mm FWHM isotropic Gaussian kernel (final smoothness of approximately $12 \times 13 \times 12 \mathrm{~mm}$ ).

\section{fMRI Data Analysis}

Following preprocessing, statistical analyses were first conducted at the individual participant level. We performed two different analyses: first, to investigate the effects of stimulus-type on visual discrimination and second, to determine whether these stimulus effects were modulated by long-term memory encoding (as defined by correct recognition memory). These analyses were restricted to correct oddity trials only. For the first analysis (Analysis 1), we modeled each condition regardless of subsequent memory, resulting in five conditions of interest (Familiar Faces, Unfamiliar Faces, Familiar Objects, Unfamiliar Objects, and Size). For the second analysis (Analysis 2), we used the 
postscanning measure of recognition memory to back-sort activity for the individual discrimination trials according to how well the item was encoded. Because of limited numbers of trials for some of the confidence levels, we followed previous fMRI studies of subsequent memory effects that have split each trial according to whether the item was later recognized with a high confidence (a " 4 " response) or later forgotten/weakly recognized ("1-3" responses; see also Behavioral Results). This comparison of items strongly confidently recognized compared with all other items is the contrast that previous studies have found most sensitive to subsequent memory effects (Otten, Henson, \& Rugg, 2001; Brewer, Zhao, Desmond, Glover, \& Gabrieli, 1998; Wagner et al., 1998). We note, however, that such a contrast may be biased toward a certain type of memory (e.g., strong memory or recollection), and it remains possible that any residual activations reflect a weaker form of memory (e.g., familiarity encoding). Because there was no memory test for unfamiliar objects or squares, Analysis 2 produced eight conditions of interest: Remembered Familiar Faces, Forgotten/Weakly Recognized Familiar Faces, Remembered Unfamiliar Faces, Forgotten/Weakly Recognized Unfamiliar Faces, Remembered Familiar Objects, Forgotten/Weakly Recognized Familiar Objects, Unfamiliar Objects, and Size. Given the different numbers of remembered and forgotten trials across sessions, the average parameter estimate for each condition was weighted by the number of trials of that condition within each session. This "weighted mean" is the standard, maximum likelihood estimate for independent samples and is more precise than the simple (unweighted) mean. The weighting was only within conditions-not across conditions-so that the sum of weights was identical for all conditions. Thus, any differences between conditions were not biased by any differences in the total number of trials per condition.

In both of these analyses, every trial was modeled using a regressor made from convolving an on-off boxcar function with a canonical hemodynamic response function, as described in Friston et al. (1998). The duration of each boxcar was equal to the stimulus duration (i.e., $5.5 \mathrm{sec}$ ). Incorrect trials and trials for which the participant failed to make a response were modeled separately as conditions of no interest. Thus, although the stimuli were presented in blocks of three items, each trial was modeled separately so that incorrect trials could be excluded from the effects of interest. To account for residual artifacts after realignment, an additional regressor was added for each volume during which excessive movement occurred (effectively discounting that volume from the effects of interest; Lemieux, Salek-Haddadi, Lund, Laufs, \& Carmichael, 2007). Excessive movement was defined as a translation of more than $0.3 \mathrm{~mm}$ in $x, y$, or $z$ directions or a rotation greater than $\pi / 90$ radians about any of the three axes, relative to the previous volume. The mean number of movement-related regressors was 3.5 per session $($ median $=0$, standard deviation $=$ 9.4). The resulting regressors, plus a constant term for each session, formed the design matrix of a general linear model.
Voxel-wise parameter estimates for these regressors were obtained by restricted maximum likelihood estimation, using a temporal high-pass filter (cutoff, $128 \mathrm{sec}$ ) to remove low-frequency drifts and modeling temporal autocorrelation across scans with an AR(1) process. Contrast images were then calculated by averaging the parameter estimates for each condition across sessions (see above).

Second-level group analyses were conducted by entering these contrast images for each participant into a single general linear model, which treated participants as a random effect. There were five conditions for Analysis 1 and eight conditions for Analysis 2. Within each model, two main $t$ contrasts were performed at every voxel (see Planned Comparisons in Results section), using a single error estimate pooled across conditions (Henson \& Penny, 2003), whose nonsphericity was estimated using restricted maximum likelihood, as described in Friston et al. (2002). SPMs of the resulting $t$ statistic were thresholded after correction for multiple comparisons to a family-wise error (FWE) of $p<$ .05 , using random field theory (Worsley et al., 1995). For the MTL and other ROIs, these corrections were applied over volumes defined by anatomical ROIs (see below); for regions outside the MTL, the correction was applied over the whole volume. When more than one suprathreshold voxel appeared for a given contrast within a given brain region, we report the coordinate with the highest $Z$ score. We report multiple coordinates for a given brain region only when they are separated by a Euclidean distance of more than the 8-mm smoothing kernel. All reported stereotactic coordinates correspond to the MNI template.

\section{ROI Analyses}

The ROIs for the above small-volume corrections were defined by anatomical masks in MNI space. Guided by a recent study in patients with temporal lobe lesions (Barense, Rogers, et al., 2010), we focused on three primary anatomical ROIs: bilateral perirhinal cortex, bilateral hippocampi, and bilateral temporal pole. The perirhinal ROI was the probability map created by Devlin and Price (2007; available at joedevlin.psychol.ucl.ac.uk/perirhinal.php). We included areas which had at least a 30\% or more probability of being perirhinal cortex. The hippocampus and temporal pole (superior and middle temporal gyri combined) ROIs were defined based on the anatomical automatic labeling atlas (Tzourio-Mazoyer et al., 2002). Further functional (based on previous data) ROI analyses were conducted on the parahippocampal place area and fusiform face area, which are reported in the Supplementary Material. All ROIs were bilateral.

\section{RESULTS}

To investigate our predictions, two different sets of smallvolume correction analyses were conducted to examine the effects of stimulus-type on visual discrimination and whether these stimulus effects were modulated by long-term memory 
encoding (as defined by correct recognition memory). These were performed on our three anatomical ROIs (see above). Each analysis had a specific set of planned comparisons described below. All contrasts were corrected for multiple comparisons to an FWE of $p<.05$, using random field theory (Worsley et al., 1995). For the perirhinal, hippocampal, and temporal pole ROIs, the minimum $Z$ score required to survive the $p<.05$ (FWE-corrected for multiple comparisons) threshold was 3.0, 3.4, and 3.6, respectively. Given our directional hypotheses, all $t$ tests were one-tailed unless stated otherwise.

\section{Analysis 1: Effects of Stimulus-type on Visual Discrimination}

We performed two planned comparisons to determine if activity in our ROIs was modulated by stimulus meaningfulness: (1) Familiar Faces-Unfamiliar Faces and (2) Familiar Objects-Unfamiliar Objects. To determine if this study replicated previous work (Barense, Henson, et al., 2010; Lee et al., 2008; Devlin \& Price, 2007), we also performed $t$ tests to compare activity for each condition relative to size oddity baseline. Finally, we performed a series of orthogonal, post hoc contrasts to investigate differences between faces versus objects (collapsing across familiarity) on the voxels identified by the above contrasts.

\section{Analysis 2: Effects of Subsequent Memory on Visual Discrimination}

To determine if the above results were modulated entirely by long-term memory encoding rather than perceptual processes, we conducted a second series of planned comparisons examining activity during the oddity judgments as a function of subsequent memory strength. To compare strongly confident memory with all other cases, we sorted trials according to stimuli that were subsequently strongly remembered (Memory Confidence Level 4) and to stimuli that were subsequently not well remembered (Memory Confidence Levels 1-3) for each stimulus type (see Methods). To check first that there were such memory effects in our ROIs, we compared activity for stimuli that were strongly remembered versus those that were not strongly remembered within each stimulus type. We then performed two planned comparisons to investigate whether effects of stimulus meaningfulness still persisted when the analysis was restricted to stimuli that were not well remembered: (1) Forgotten/Weakly Recognized Familiar Faces-Forgotten/Weakly Recognized Unfamiliar Faces and (2) Forgotten/Weakly Recognized Familiar ObjectsUnfamiliar Objects (because of floor performance during piloting, there was no memory test for unfamiliar objects). To determine whether any observed regions of activity were spatially equivalent to those from Analysis 1, we compared the Euclidean distance of any suprathreshold voxels from Analysis 2 to the voxels revealed by the corresponding comparisons of Analysis 1. Unless otherwise noted, this distance was less than the estimated final smoothness.

\section{Behavioral Data}

The accuracy and RT data are shown in Table 1. As planned from extensive piloting, there was no significant difference in accuracy between Familiar Faces and Unfamiliar Faces or between Familiar Objects and Unfamiliar Objects $(t \mathrm{~s}<1.1$, $p s>.3$, two-tailed), suggesting that the fMRI data for our critical comparisons are unlikely to be confounded by difficulty. When compared with the size baseline, there was no significant difference in accuracy for Unfamiliar Faces, Familiar Objects, and Unfamiliar Objects ( $t \mathrm{~s}<1.6, p \mathrm{~s}>.1$, two-tailed), but there was a significant difference for Familiar Faces $(t(17)=2.54, p<.05$, two-tailed), with Familiar Faces being significantly easier than the size baseline. In terms of RTs, familiar face discriminations were solved significantly faster than unfamiliar face discriminations, as were familiar object discriminations compared with unfamiliar object discriminations ( $t \mathrm{~s}>3.9, p \mathrm{~s}<.01$, two-tailed). The size discriminations were solved significantly faster than all face and object discriminations ( $t \mathrm{~s}>6.3, p s<.001$, two-tailed). Despite these significant RT differences, we think it unlikely that our imaging data can be explained solely by differences in time spent on each task (see Discussion).

The mean proportions of responses for each memory judgment and stimulus type during the postscanning surprise memory test are shown in Table 2 . We also calculated the corrected recognition score ( $\mathrm{Pr}$; proportion of hits proportion of false alarms) collapsed across Confidence Levels 3 and 4 (i.e., all items that were labeled as old). Pr was significantly greater than zero for all three conditions (Familiar Faces, Familiar Objects, and Unfamiliar Faces; $t$ s $>$ 15.9 , ps < .001). We also found that memory was better for Familiar Faces and Familiar Objects compared with Unfamiliar Faces $(t \mathrm{~s}>5.0, p \mathrm{~s}<.001)$ and that memory for Familiar Objects was better than memory for Familiar Faces $(t \mathrm{~s}>3.0, p s<.01)$.

\section{Imaging Data}

Analysis 1: Effects of Stimulus-type on Visual Discrimination

Perirbinal ROI. The planned contrasts to investigate effects of stimulus meaningfulness revealed significant

Table 1. Mean Accuracy and RT (Correct Trials Only) Scores for Each Condition (Standard Deviations Shown in Parentheses)

\begin{tabular}{lccccc}
\hline & Familiar Faces & Unfamiliar Faces & Familiar Objects & Unfamiliar Objects & Size \\
\hline Proportion correct & $0.87(0.07)$ & $0.84(0.07)$ & $0.77(0.07)$ & $0.77(0.09)$ & $0.79(0.10)$ \\
RT $(\mathrm{msec})$ & $2616(415)$ & $2796(349)$ & $3009(448)$ & $3300(386)$ & $2110(366)$ \\
\hline
\end{tabular}


Table 2. Recognition Memory Performance: Mean Proportion of Responses across Participants to Old and New Items of Each Stimulus Type (Standard Deviations Shown in Parentheses)

\begin{tabular}{cccc}
\hline \multicolumn{4}{c}{ Recognition Judgments } \\
\cline { 2 - 4 } & 3 & 2 & 1 \\
\hline
\end{tabular}

Familiar Faces

$\begin{array}{lllll}\text { Old } & 0.42(0.14) & 0.23(0.09) & 0.22(0.10) & 0.11(0.10) \\ \text { New } & 0.06(0.03) & 0.14(0.09) & 0.31(0.11) & 0.46(0.17)\end{array}$

Unfamiliar Faces

$\begin{array}{lllll}\text { Old } & 0.11(0.07) & 0.31(0.10) & 0.44(0.15) & 0.11(0.09) \\ \text { New } & 0.02(0.02) & 0.14(0.08) & 0.55(0.19) & 0.26(0.18)\end{array}$

Familiar Objects

\begin{tabular}{lllll} 
Old & $0.56(0.14)$ & $0.19(0.10)$ & $0.10(0.04)$ & $0.12(0.10)$ \\
New & $0.07(0.06)$ & $0.11(0.10)$ & $0.20(0.11)$ & $0.59(0.19)$ \\
\hline
\end{tabular}

$1=$ definitely new; 2 = somewhat sure it is new; $3=$ somewhat sure it is old 4 = definitely old .

activity in perirhinal cortex for both faces and objects. For faces, the contrast of Familiar Faces-Unfamiliar Faces revealed suprathreshold activity in left perirhinal cortex $[(-33-12-27 ; Z=3.6),(-30-6-30 ; Z=3.0)]$. For objects, the contrast of Familiar Objects-Unfamiliar Objects revealed bilateral activity in perirhinal cortex $[(-30$ $-18-27 ; Z=4.4) ;(36-9-30 ; Z=3.6)]$. When activity was collapsed across familiarity levels, none of these voxels showed preferential activity for either faces or objects (i.e., there was no main effect of stimulus domain in these voxels). Relative to the size baseline, we observed suprathreshold bilateral perirhinal activity for all four conditions (all $Z \mathrm{~s}>$ 3.7). Representative voxels are shown in Figure $2 \mathrm{~A}$ and $\mathrm{B}$.

Hippocampal ROI. The planned contrasts to investigate effects of stimulus meaningfulness revealed significant bilateral activity in the anterior hippocampus for faces and a more posterior hippocampal region for objects. For faces, the contrast of Familiar Faces-Unfamiliar Faces revealed suprathreshold activity in left $[(-21-9-18 ; Z=5.1)$, $(-30-6-18 ; Z=5.1)]$ and right $[27-15-18 ; Z=$ 4.9] anterior hippocampus. When activity was collapsed across familiarity levels, all of these above voxels demonstrated more activity for faces than for objects (i.e., a main effect of Faces $>$ Objects; $Z s>4.6$ ). Representative voxels are shown in Figure 2C and D.

For objects, the contrast of Familiar Objects-Unfamiliar Objects revealed bilateral local maxima more posteriorly at the boundary of the hippocampus and parahippocampal cortex $[(33-33-12 ; Z=7.3) ;(33-21-21 ; Z=7.2)$; $(-33-27-15 ; Z=6.9) ;(-30-33-9 ; Z=5.5)]$. When activity was collapsed across familiarity levels, all of these voxels demonstrated significantly more activity for objects than for faces ${ }^{1}(Z s>3.3)$. Representative voxels are shown in Figure 2E and F. Relative to the size baseline, we observed suprathreshold bilateral hippocampal activity for all four conditions (all $Z s$ > 5.3).

Temporal pole ROI. The planned contrasts to investigate effects of stimulus meaningfulness revealed activity in the temporal pole for faces but not objects. For faces, the contrast of Familiar Faces-Unfamiliar Faces revealed suprathreshold activity in the left temporal pole $[(-36$ $18-27 ; Z=4.5) ;(-273-21 ; Z=3.9)]$ and marginal activity in the right temporal pole $[633-18 ; Z=3.5, p=.06$, FWE-corrected]. When activity was collapsed across familiarity levels, we found significantly more activity for faces compared with objects in the right temporal voxel $(Z=4.4)$. Relative to the size baseline, we observed suprathreshold bilateral temporal pole activity for both familiar and unfamiliar face conditions ( $Z$ 's $>4.1$ ), but not for either the familiar or unfamiliar object conditions. Representative voxels are shown in Figure $2 \mathrm{G}$ and $\mathrm{H}$.

The reverse familiarity contrasts for faces and objects [i.e., (Unfamiliar Faces-Familiar Faces) and (Unfamiliar ObjectsFamiliar Objects)] revealed no suprathreshold voxels in any of the three ROIs.

\section{Analysis 2: Subsequent Memory Analysis}

We first performed an analysis comparing activity observed during discriminations for subsequently strongly remembered stimuli (Memory Confidence Level 4) to subsequently weakly remembered/forgotten stimuli (Memory Confidence Levels 1-3) for each stimulus type (see description of Analysis 2 in Methods). We observed significant activity for familiar faces in the left perirhinal cortex $[-36-15-27 ; Z=3.3]$ and left hippocampus [ $-36-12$ $-24 ; Z=3.3]$. For objects, we observed significant memory effects in the left perirhinal cortex [30 $6-27 ; Z=$ 3.0]. These voxels are shown in Figure 3A-C.

Figure 2. Effects of stimulus type. fMRI signal change relative to size baseline (i.e., size oddity was subtracted from each condition) in suprathreshold voxels from the three ROIs in Analysis 1. Significance is shown for the comparisons of familiar versus unfamiliar within each stimulus type (as indicated by arrows) and the comparison of a given condition relative to size baseline (as indicated by an asterisk above the bar): $* p<.05$ (FWE-corrected), $* * p<.01$ (FWE-corrected), $\diamond p=.06$ (FWE-corrected). Error bars represent $S E M$ of difference between each condition and the baseline condition. 


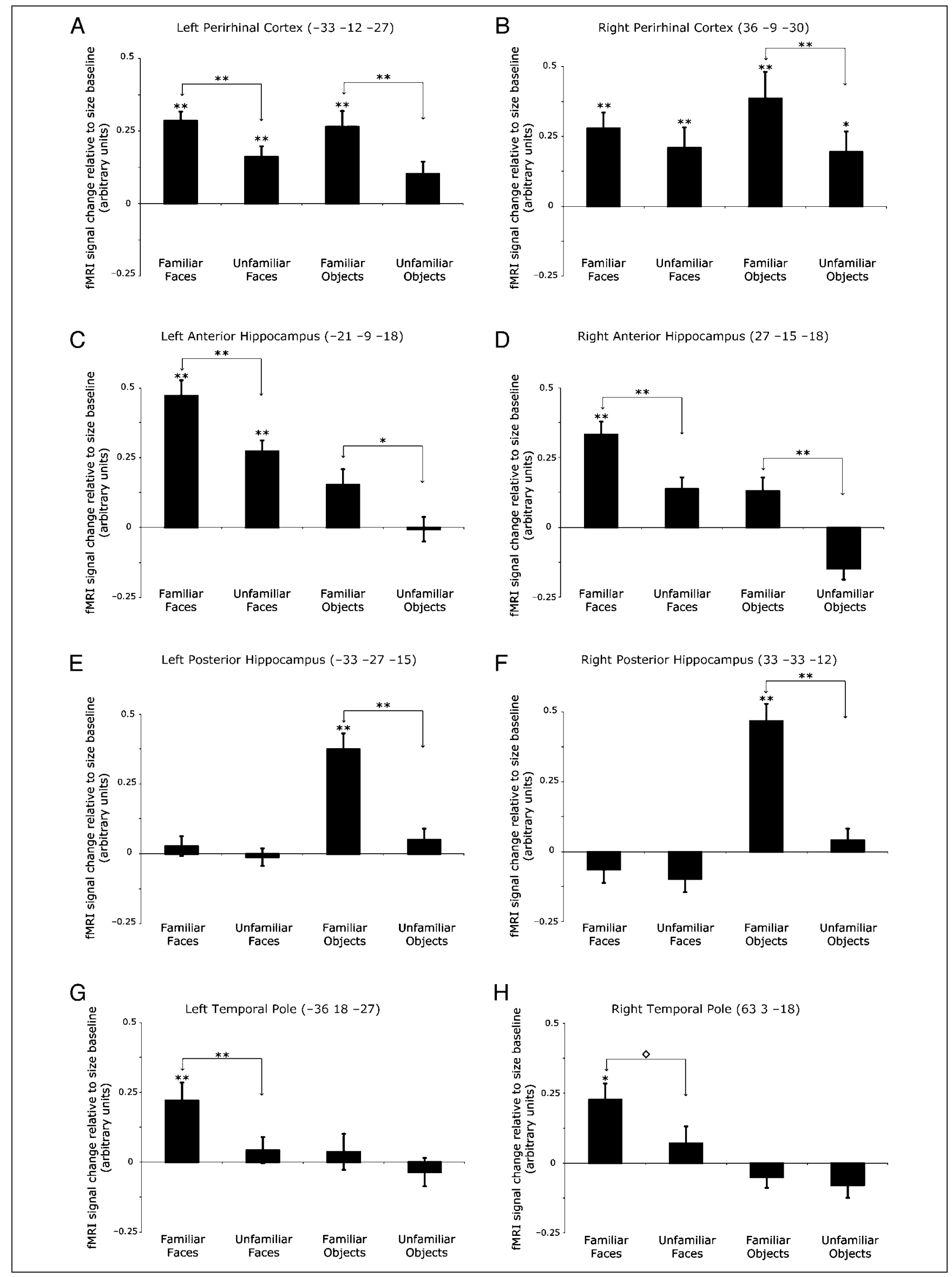




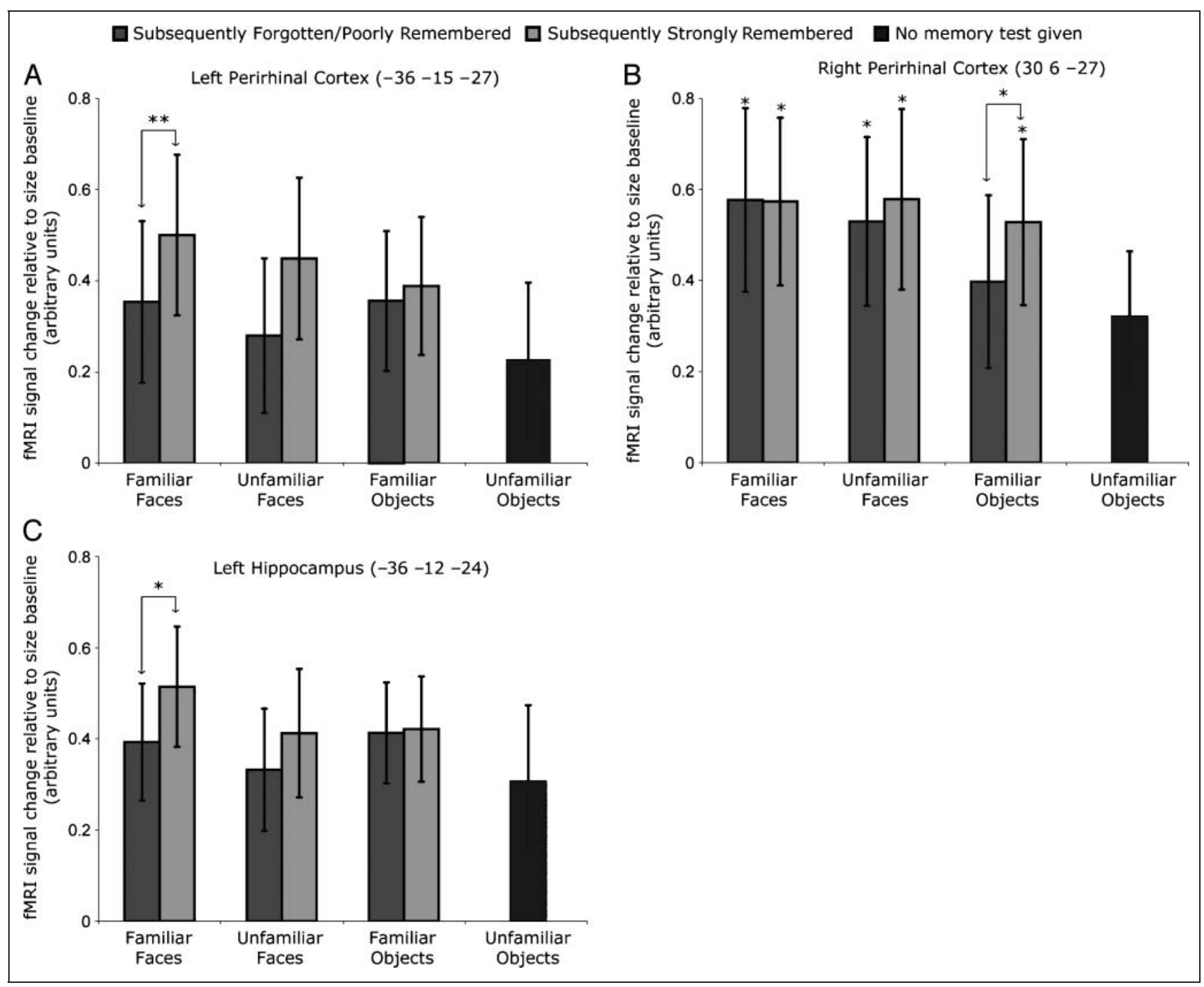

Figure 3. Significant memory effects. fMRI signal change relative to size baseline (i.e., size oddity was subtracted from each condition) in suprathreshold voxels from the three ROIs for the contrast of forgotten stimuli versus well-remembered stimuli. * $p<.05$ (FWE-corrected), $* * p<.01$ (FWE-corrected). Error bars represent SEM of difference between each condition and the baseline condition.

Perirbinal ROI. The planned contrasts revealed a trend toward significant activity ( $p=.08$, FWE-corrected) for Forgotten/Weakly Recognized Familiar Faces relative to Forgotten/Weakly Recognized Unfamiliar Faces in two left perirhinal voxels $[(-33-12-27 ; Z=2.8) ;(-36-6$ $-30 ; Z=2.8)]$.

For the contrast of Forgotten/Weakly Recognized Familiar Objects-Unfamiliar Objects, there was a significant voxel at the boundary of the right perirhinal cortex and temporal pole [36 15 -27; $Z=3.8$ ] (this voxel was not observed in Analysis 1). Relative to the size baseline, we observed significant left perirhinal activity for Forgotten/Weakly Rec- ognized stimuli in all four conditions $(Z s>3.5)$. The Unfamiliar Object condition was the only condition to produce suprathreshold activity in the right perirhinal cortex $(33-6-30 ; Z=3.6)$.

Hippocampal ROI. For faces, the planned contrast to investigate effects of stimulus meaningfulness in stimuli that were not well remembered revealed significantly activity in the hippocampus bilaterally $[(-15-6-15 ; Z=4.5) ;(-21$ $-9-15 ; Z=4.1) ;(-27-21-15 ; Z=4.0) ;(24-12-18$; $Z=4.0)]$. Representative voxels are shown in Figure 4C and $\mathrm{D}$.

Figure 4. Effects of subsequent memory. fMRI signal change relative to size baseline (i.e., size oddity was subtracted from each condition) in suprathreshold voxels from the three ROIs in Analysis 2. Significance is shown for the comparisons of familiar versus unfamiliar within each stimulus type (as indicated by arrows) and the comparison of a given condition relative to size baseline (as indicated by an asterisk above the bar): $+p<.08$ (FWE-corrected), $* p<.05$ (FWE-corrected), $* * p<.01$ (FWE-corrected). Error bars represent SEM of difference between each condition and the baseline condition. 
口Subsequently Forgotten/Poorly Remembered $\boldsymbol{\square}$ Subsequently Strongly Remembered $\mathbf{a}$ No memory test given
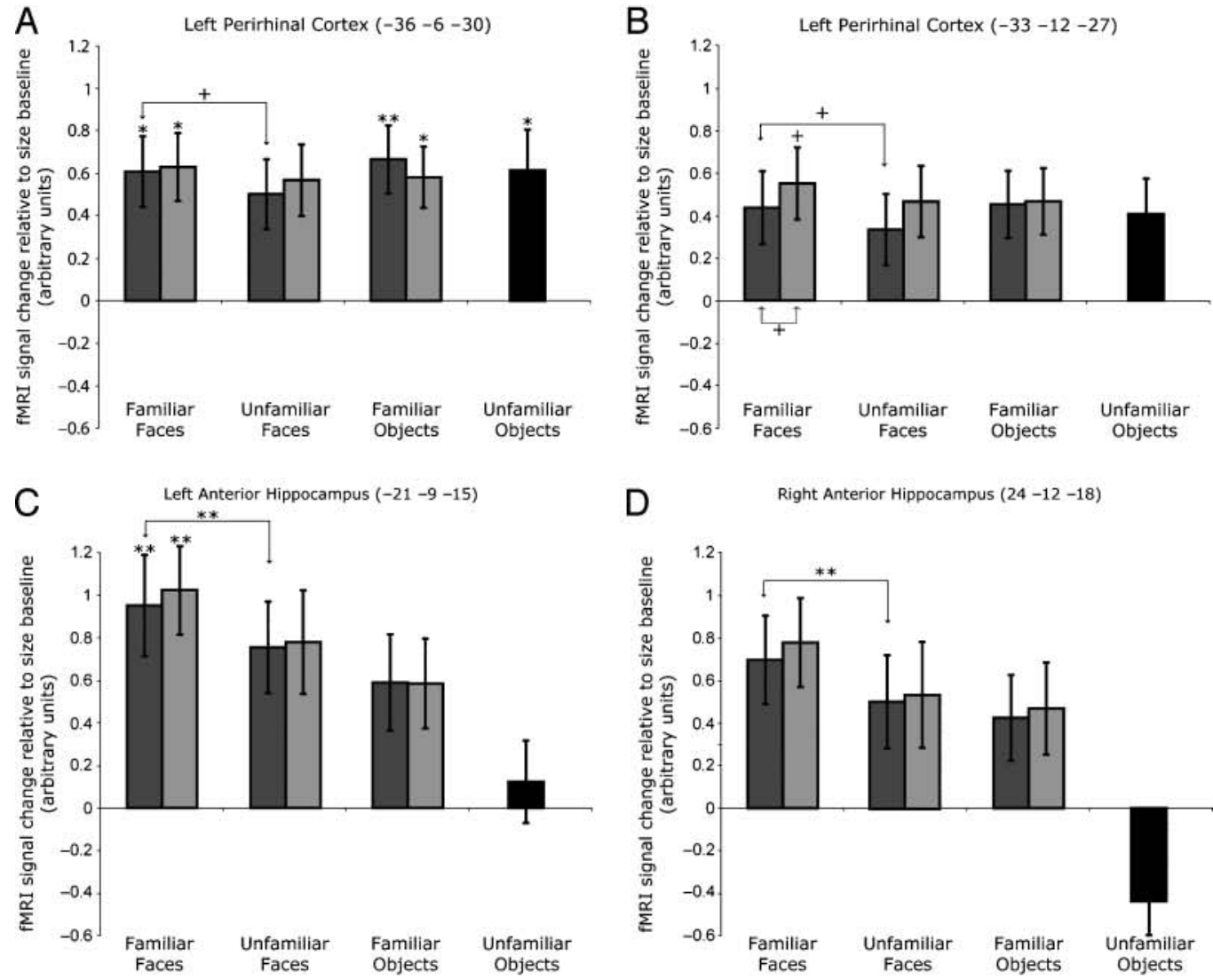

Dight Anterior Hippocampus $(24-12-18)$

E

Left Posterior Hippocampus (-27 -24 -15)
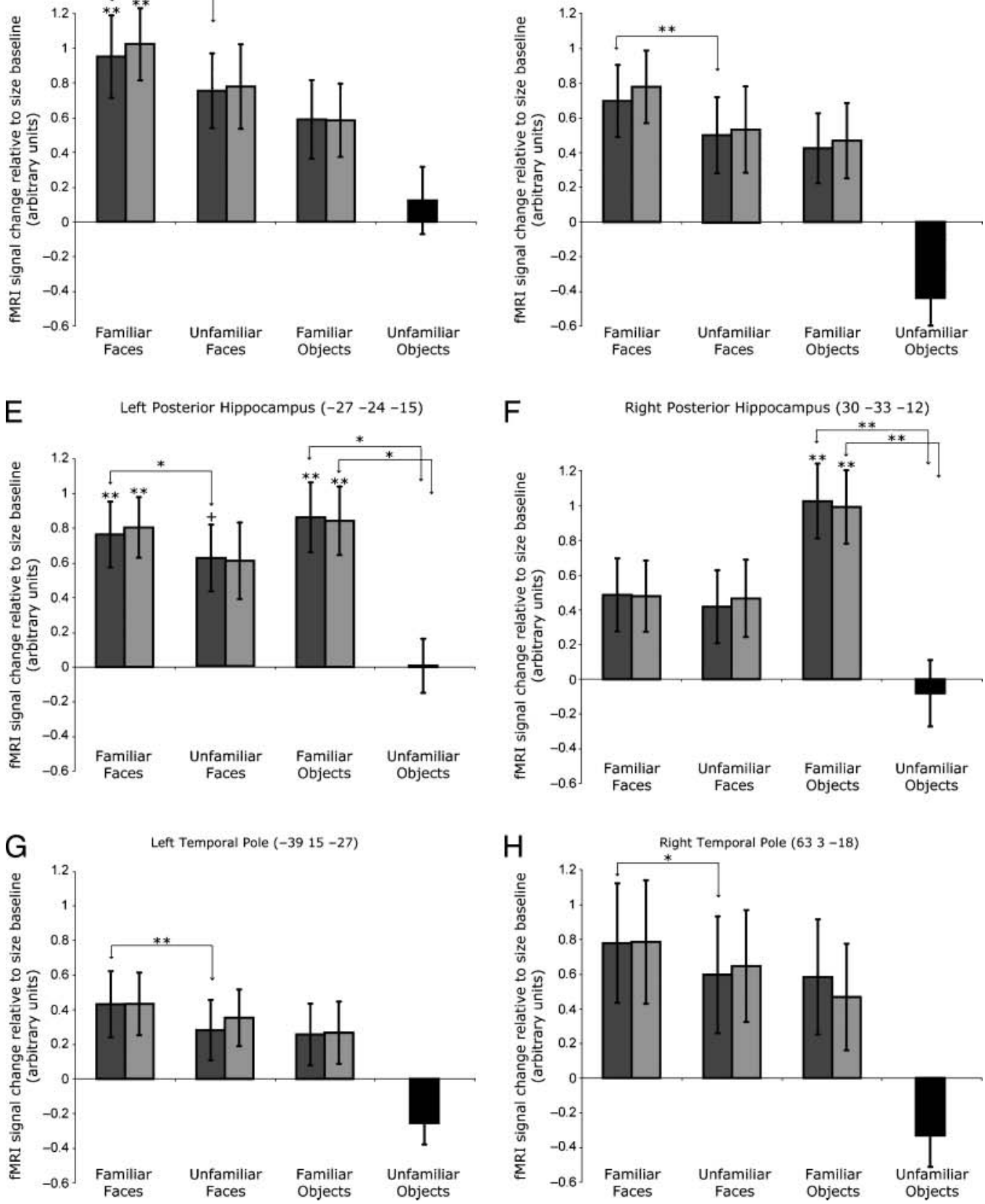


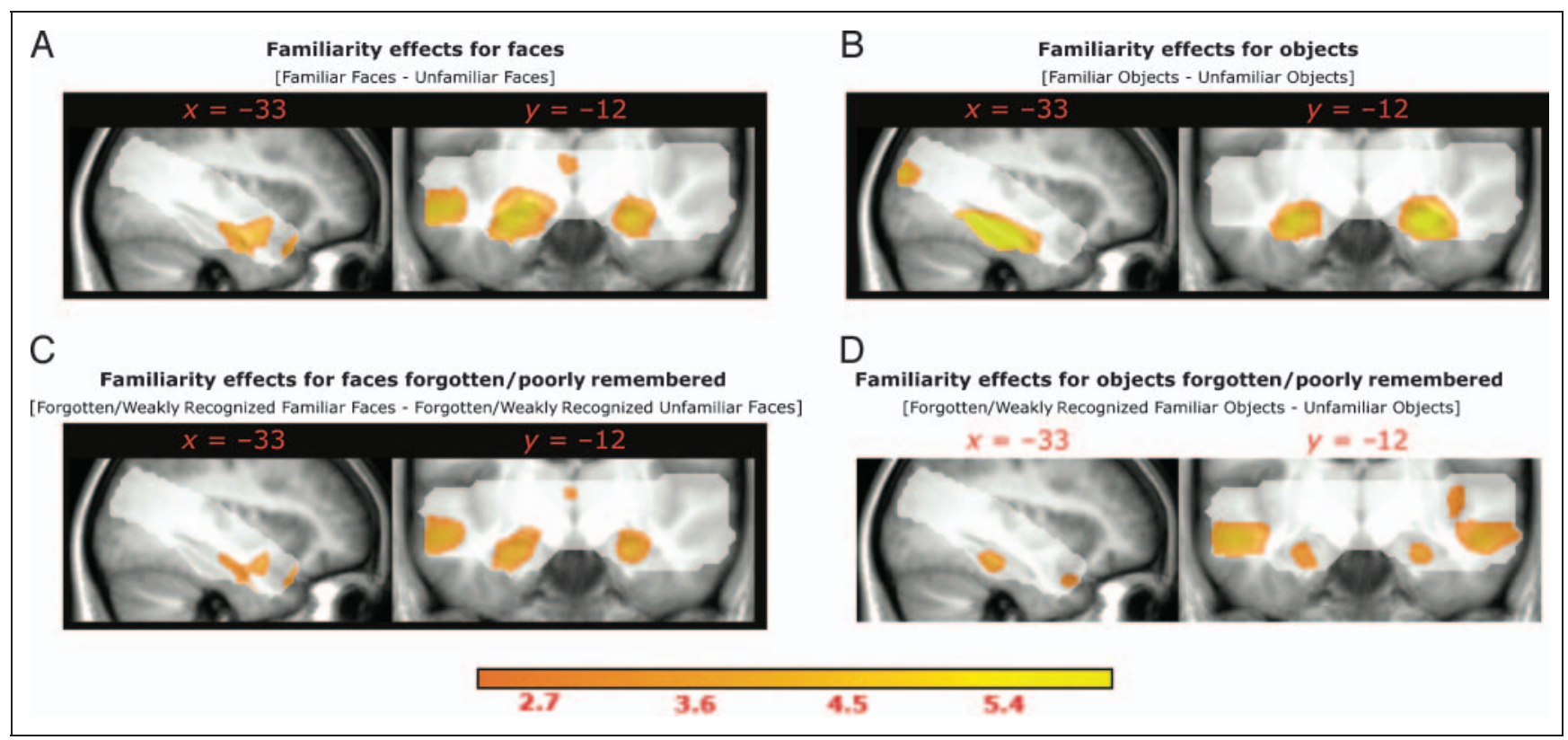

Figure 5. Voxels showing effects of each of the two, a priori contrasts from Analyses 1 to 2 across the entire image. To show the spatial extent of the activations, these maps were thresholded at $p<.01$ (uncorrected). Statistical maps are superimposed on the mean structural image for all participants in the present study. To show regions of signal dropout, the mask image across all participants at the second level is also superimposed (in white). The color bar reflects $t$ values.

For objects, bilateral local maxima were observed more posteriorly at the boundary of the hippocampus and parahippocampal cortex $[(27-27-15 ; Z=4.6) ;(30-33-12$; $Z=3.6) ;(-27-24-15 ; Z=3.6) ;(-27-33-9 ; Z=3.3$, $p=.08)]$. Relative to the size baseline, we observed significant bilateral hippocampal activity for Forgotten/Weakly Recognized stimuli in all four conditions $(Z s>4.7)$. Representative voxels are shown in Figure $4 \mathrm{E}$ and $\mathrm{F}$.

Temporal pole ROI. The planned comparisons to investigate stimulus meaningfulness in stimuli that were not well remembered revealed local maxima in the temporal pole bilaterally for faces $[(-3915-27 ; Z=4.1) ;(-4818-18$; $Z=4.1) ;(633-18 ; Z=3.5)]$ and for objects $[(543-18$; $Z=5.2) ;(-573-12 ; Z=4.4)]$ (these local maxima for objects were not observed in Analysis 1). Relative to the size baseline, we observed significant bilateral temporal pole activity for Forgotten/Weakly Recognized Familiar and Unfamiliar Faces $(Z s>4.0)$ and significant left temporal pole activity for Forgotten/Weakly Recognized Familiar Objects (54 $3-18 ; Z=4.0$ ). Representative voxels are shown in Figure $4 \mathrm{G}$ and $\mathrm{H}$.

The reverse contrasts for faces and objects [i.e., (Forgotten/ Weakly Recognized Unfamiliar Faces-Forgotten/Weakly Recognized Familiar Faces) and (Forgotten/Weakly Recognized Unfamiliar Objects-Forgotten/Weakly Recognized Familiar Objects)] revealed no suprathreshold voxels in any of the three ROIs.

As one of the aims of the study was to determine if activity observed during perceptual discrimination (Analysis 1) could be explained entirely by memory encoding, Analy- sis 2 focused on stimuli that were not well remembered. It is important to note, however, that when we performed the same comparisons described above considering only stimuli that were subsequently strongly remembered (Memory Confidence Level 4), we found suprathreshold local maxima in all of the above ROIs for both faces and objects, indicating that these effects persist regardless of subsequent memory status.

Whole-image analysis. The same two planned comparisons for both Analyses 1 and 2 were also performed on a voxel-by-voxel basis to investigate brain regions outside the MTL showing any effects of stimulus type. For maxima outside the MTL, a threshold of $p<.05$, two-tailed and FWE-corrected for the whole brain was applied. The results for faces and objects are listed in Supplementary Tables 1-7.

To show the spatial extent of all activations, Figure 5 displays statistical maps across the whole image for each of the two planned comparisons of Analyses 1 and 2 using a liberal threshold of $p<.01$ uncorrected. Statistical maps are superimposed on the mean structural image for all participants in the present study.

\section{DISCUSSION}

Using a visual discrimination paradigm sensitive to temporal lobe damage in humans (Barense, Rogers, et al., 2010; Lee, Buckley, et al., 2005) and monkeys (Buckley et al., 2001), the present investigation sought to identify which temporal lobe regions were modulated by face and object familiarity during 
perceptual discrimination. Discriminations involving familiar (i.e., famous) faces, relative to unfamiliar faces, activated regions in the perirhinal cortex, temporal pole, and hippocampus. Discriminations involving familiar everyday objects, relative to novel objects (greebles), activated regions in the perirhinal cortex and hippocampus. There was no overt requirement for participants to either name or identify the faces and objects, and thus, these activation differences reflect more automatic or incidental conceptual processing and semantic retrieval about the items. Moreover, these differences do not reflect solely incidental long-term memory encoding, as they persisted for faces (in all structures) and objects (in the hippocampus) that were subsequently not well remembered. The results provide further evidence that the MTL is critical for complex perceptual discriminations, and additionally suggest that these structures are recruited during semantic processing of objects and faces.

These findings are consistent with the theory that the perirhinal cortex and neighboring anteromedial-temporal structures function as the endpoint of the representational hierarchy within the ventral visual stream, supporting finegrained discriminations among objects and faces (e.g., Baxter, 2009; O’Neil et al., 2009; Barense et al., 2007; Bussey \& Saksida, 2007; Lee, Barense, \& Graham, 2005). We observed above-baseline activity for these complex discriminations in all our anatomical ROIs - for both familiar and novel stimuli. The additional observation, however, that these regions showed more activity for familiar, relative to novel, stimuli, suggests that this same system is also involved in processing conceptual information about objects and faces. This is consistent with findings that the perirhinal cortex is critical for the cross-modal integration of meaningful object features (Holdstock et al., 2009; Taylor et al., 2009) and suggests that anterior temporal lobe structures act as an interface between perceptual processing and conceptual knowledge.

The finding of increased temporal pole activity for famous, relative to novel, faces is in line with previous work (Gorno-Tempini et al., 1998, 2001; Leveroni et al., 2000; Damasio, Grabowski, Tranel, Hichwa, \& Damasio, 1996; Sergent, Ohta, \& MacDonald, 1992). These findings have led to a theory that the temporal pole is critical for knowledge of semantically unique items - that is, items that have a one-to-one relationship with their referents. There are few categories other than familiar faces that have such unique semantic associations, but some studies designed to address this idea found that famous buildings or landmarks (which are semantically unique) also implicated the temporal pole (Gorno-Tempini et al., 2001; Grabowski et al., 2001; Nakamura et al., 2000). Another account holds that the temporal pole is critical for processing social conceptual information (such attributing thoughts, beliefs, or intentions to others) and that this more general involvement in social semantic memory underlies the increased activity observed for familiar faces (e.g., Ross \& Olson, 2010; Simmons, Reddish, Bellgowan, \& Martin, 2010; Zahn et al., 2009; Olson, Plotzker, \& Ezzyat, 2007; Zahn et al., 2007).
The present results are consistent with either of these hypotheses.

We also observed above-baseline activity and significant familiarity effects in the hippocampus. This is inconsistent with the intact performance of patients with selective hippocampal lesions on nearly identical oddity discriminations involving familiar and novel objects (Barense et al., 2007) and unfamiliar faces (Lee, Buckley, et al., 2005) but consistent with our previous fMRI findings of above baseline activity for oddity discriminations of novel objects and faces (Barense, Henson, et al., 2010). One possibility is that this hippocampal activity reflects a hippocampal comparator mechanism (i.e., a match-mismatch signal) underlying associative novelty detection (Kumaran \& Maguire, 2006a, 2006b, 2007). It is possible that such a mechanism was operating in the present study - as a participant compared different stimuli within a trial, the hippocampus signaled a mismatch to the odd-one-out. However, given the intact performance of patients with hippocampal damage on these oddity tasks, although this activity may be automatic, it does not appear necessary to support normal levels of performance. Another possibility is that the hippocampal activity reflected incidental retrieval of pre-experimental personal knowledge about the faces and objects presented. A recent study demonstrated that hippocampal activity increased with how well participants knew the person depicted during a recognition memory test (either famous or personally known; Trinkler, King, Doeller, Rugg, \& Burgess, 2009). This activity was observed during both study and test portions of the recognition memory test and was not modulated by how recently the familiar face had been presented (i.e., activity was observed during both hits and correct rejections), suggesting that the hippocampal activity reflected incidental pre-experimental personally relevant knowledge about the person presented.

This study was originally inspired by the finding that two groups of patients with temporal lobe lesions (densely amnesic cases with focal lesions to the MTL and patients with $\mathrm{SD})$ demonstrated strikingly different influences of stimulus meaningfulness on discriminations involving novel and familiar objects (Barense, Rogers, et al., 2010). Although both groups of patients were impaired on both novel and familiar discriminations, performance in the MTL amnesics was facilitated by the use of familiar objects, whereas patients with SD showed no benefit from the use of meaningful objects. These findings suggest that the MTL cases were able to engage semantic support that was presumably not available to the SD patients (see also MacKay \& James, 2009; MacKay et al., 2008; Moses et al., 2008). The present study indicates that the perirhinal cortex, hippocampus, and temporal pole all contribute to semantic processing during simple perceptual discriminations and are possible neuroanatomical loci for this semantic support. The finding that the MTL cases - who also had extensive damage to the perirhinal cortex, hippocampus, and temporal poleshowed facilitation from the use familiar stimuli, however, poses a bit of a puzzle. One possible explanation is that 
the MTL patients temporal pole damage was more lateralized to the right (Barense, Rogers, et al., 2010; Lee \& Rudebeck, 2010), whereas temporal pole damage in SD tends to affect primarily the left temporal lobe early in the disease (e.g., Rosen et al., 2002; Mummery et al., 2000). Another possibility is that anterolateral temporal lobe regions, which are damaged in SD but not in the MTL amnesics, contributed to the semantic facilitation (Levy, Bayley, \& Squire, 2004; Chan et al., 2001; Galton et al., 2001). Consistent with this idea, we found above threshold activity in anterolateral temporal regions (BA 20/21) for familiar relative to novel discriminations (see Figure 5 and Supplementary Material). Finally, it is also possible that these behavioral differences between patient groups may reflect differences in the nature of their pathology. Recent neuropsychological studies, complemented with computational modeling, have demonstrated that different etiologies (e.g., neurodegenerative versus encephalitic) can result in different profiles of impairment on semantic tasks. The underlying causes of these patterns are thought to be separate: when semantic representations are degraded or "dimmed" (fewer connections available) a global, generalized semantic impairments emerge, as in SD. By contrast, when the representations are distorted (important weights removed), a category-specific pattern of deficits appears, as in viral encephalitis (Lambon Ralph, Lowe, \& Rogers, 2007).

In addition to investigating effects of stimulus familiarity, we also compared effects of stimulus domain (i.e., faces versus objects). When we collapsed across familiarity and compared activity for faces relative to objects in suprathreshold voxels identified by the familiarity contrasts, we found that anterior hippocampal voxels were face preferential whereas more posterior hippocampal/parahippocampal voxels were object preferential. In the temporal pole, we found face preferential but not object preferential voxels. In the perirhinal cortex, we found neither face- nor object preferential voxels. There is an emerging body of literature investigating category selectivity in the MTL (e.g., Barense, Henson, et al., 2010; Preston et al., 2010; Bellgowan, Buffalo, Bodurka, \& Martin, 2009; Litman, Awipi, \& Davachi, 2009; Mundy et al., 2009; Diana, Yonelinas, \& Ranganath, 2008; Lee et al., 2008), with many of the investigations focusing on comparisons between scenes, objects, and faces. For example, Litman et al. (2009) report a representational gradient, with scene preferential responses in posterior parahippocampal and object preferential responses in anterior perirhinal cortex. Consistent with what we observed here, these authors reported that objects produced above baseline responses throughout the entire anterior-posterior extent of parahippocampal cortex, whereas faces produced above baseline activity in anterior MTL cortical regions only.

There were some significant differences in RTs across the different conditions, with the familiar conditions of both faces and objects being solved significantly faster than their corresponding unfamiliar conditions and size being solved significantly faster than all other conditions. Despite these significant RT differences, we think it unlikely that our observed activations can be explained solely by differences in time spent on each task. First, with regard to our critical comparisons of stimulus familiarity, participants take longer to respond for the unfamiliar stimuli compared with the familiar stimuli, yet we find lower levels of activity in the unfamiliar conditions. Second, when we ran a different model for the event-related responses within each participant, in which the duration of neural activity was related to the RT on each trial (rather than the fixed trial duration), the results did not change in any important way.

Finally, we note that our contrasts to investigate the effects of subsequent memory were biased toward strong memories, and the residual activity may reflect a weaker form of memory (e.g., familiarity encoding). To address this, we performed analyses to investigate activity related to each confidence level separately and found no evidence to support weaker memory effects in any of our ROIs, suggesting that activity specific to weak encoding did not drive our results. However, these analyses relied on too few trials to obtain reliable estimates of activity, and thus, we would be hesitant to draw strong claims from this null result.

In summary, the present experiment suggests that representations of complex objects and faces (dependent on MTL structures) interact with higher-order conceptual processes in the service of perceptual tasks with no overt semantic component. When we restricted our analyses to stimuli that were subsequently not well remembered, we still observed effects of stimulus familiarity and abovebaseline activity in all our ROIs, indicating that these findings cannot be attributed solely to incidental encoding into long-term memory. We do not wish to argue, however, that encoding and perception are entirely mutually exclusive processes. The idea that a memory trace reflects processes carried out primarily for the purposes of perception and that more meaningful processing leads to better memory has a long and esteemed history in cognitive psychology (Craik, 2002; Craik \& Tulving, 1975; Craik \& Lockhart, 1972). The current data are entirely consistent with the idea that the stronger the perceptual representation, the greater the likelihood of successful memory. It should not be surprising, therefore, that the same regions involved in perceiving complex stimuli are also involved in remembering them.

\section{Acknowledgments}

We thank Michael J. Tarr (Carnegie Mellon University) for providing the greeble stimuli and Christian Schwarzbauer for technical assistance. This research was funded by a Discovery Grant from the Natural Sciences and Engineering Research Council of Canada (M. D. B.), the Wales Institute of Cognitive Neuroscience (K. G.), and the UK Medical Research Council (WBSE U.1055.05.012.00001.01).

Reprint requests should be sent to Morgan D. Barense, Department of Psychology, University of Toronto, 100 St. George Street, Toronto, Ontario, M5S 3G3 Canada, or via e-mail: barense@ psych.utoronto.ca. 


\section{Note}

1. This main effect for objects versus faces was driven by the contrast of Familiar Objects-Familiar Faces (significant in all voxels, $Z s$ > 4.8), not the contrast of Unfamiliar Objects-Unfamiliar Faces (which was not significant in any of the above voxels). However, these post hoc simple effect contrasts are biased by the selection of the voxels, given that the selection contrast of Familiar Objects-Unfamiliar Objects is not orthogonal to the contrast of Familiar Objects-Familiar Faces.

\section{REFERENCES}

Barense, M. D., Bussey, T. J., Lee, A. C., Rogers, T. T., Davies, R. R., Saksida, L. M., et al. (2005). Functional specialization in the human medial temporal lobe. Journal of Neuroscience, 25, 10239-10246.

Barense, M. D., Gaffan, D., \& Graham, K. S. (2007). The human medial temporal lobe processes online representations of complex objects. Neuropsychologia, 45, 2963-2974.

Barense, M. D., Henson, R. N. A., Lee, A. C., \& Graham, K. S. (2010). Medial temporal lobe activity during complex discrimination of faces, objects, and scenes: Effects of viewpoint. Hippocampus, 20, 389-401.

Barense, M. D., Rogers, T. T., Bussey, T. J., Saksida, L. M., \& Graham, K. S. (2010). Influence of conceptual knowledge on visual object discrimination: Insights from semantic dementia and MTL amnesia. Cerebral Cortex, 20, 2568-2582.

Baxter, M. G. (2009). Involvement of medial temporal lobe structures in memory and perception. Neuron, 61, 667-677.

Bellgowan, P. S., Buffalo, E. A., Bodurka, J., \& Martin, A. (2009). Lateralized spatial and object memory encoding in entorhinal and perirhinal cortices. Learning and Memory, 16, 433-438.

Binney, R. J., Embleton, K. V., Jefferies, E., Parker, G. J., \& Ralph, M. A. (2010). The ventral and inferolateral aspects of the anterior temporal lobe are crucial in semantic memory: Evidence from a novel direct comparison of distortioncorrected fMRI, rTMS, and semantic dementia. Cerebral Cortex, 20, 2728-2738.

Brewer, J. B., Zhao, Z., Desmond, J. E., Glover, G. H., \& Gabrieli, J. D. (1998). Making memories: Brain activity that predicts how well visual experience will be remembered. Science, 281, 1185-1187.

Buckley, M. J., Booth, M. C., Rolls, E. T., \& Gaffan, D. (2001). Selective perceptual impairments after perirhinal cortex ablation. Journal of Neuroscience, 21, 9824-9836.

Bussey, T. J., \& Saksida, L. M. (2007). Memory, perception, and the ventral visual-perirhinal-hippocampal stream: Thinking outside of the boxes. Hippocampus, 17, 898-908.

Bussey, T. J., Saksida, L. M., \& Murray, E. A. (2002). Perirhinal cortex resolves feature ambiguity in complex visual discriminations. European Journal of Neuroscience, 15, 365-374.

Bussey, T. J., Saksida, L. M., \& Murray, E. A. (2003). Impairments in visual discrimination after perirhinal cortex lesions: Testing "declarative" vs. "perceptual-mnemonic" views of perirhinal cortex function. European Journal of Neuroscience, 17, 649-660.

Chan, D., Fox, N. C., Scahill, R. I., Crum, W. R., Whitwell, J. L., Leschziner, G., et al. (2001). Patterns of temporal lobe atrophy in semantic dementia and Alzheimer's disease. Annals of Neurology, 49, 433-442.

Cowell, R. A., Bussey, T. J., \& Saksida, L. M. (2010). Functional dissociations within the ventral object processing pathway: Cognitive modules or a hierarchical continuum? Journal of Cognitive Neuroscience, 22, 2460-2479.
Craik, F. I. M. (2002). Levels of processing: Past, present. and future? Memory, 10, 305-318.

Craik, F. I. M., \& Lockhart, R. S. (1972). Levels of processing: A framework for memory research. Journal of Verbal Learning and Verbal Behaviour, 11, 671-684.

Craik, F. I. M., \& Tulving, E. (1975). Depth of processing and the retention of words in episodic memory. Journal of Experimental Psychology: General, 104, 268-294.

Cusack, R., \& Papadakis, N. (2002). New robust 3-D phase unwrapping algorithms: Application to magnetic field mapping and undistorting echoplanar images. Neuroimage, 16, 754-764.

Damasio, H., Grabowski, T. J., Tranel, D., Hichwa, R. D., \& Damasio, A. R. (1996). A neural basis for lexical retrieval. Nature, 380, 499-505.

Davachi, L., \& Wagner, A. D. (2002). Hippocampal contributions to episodic encoding: Insights from relational and item-based learning. Journal of Neurophysiology, 88, 982-990.

Davies, R. R., Graham, K. S., Xuereb, J. H., Williams, G. B., \& Hodges, J. R. (2004). The human perirhinal cortex and semantic memory. European Journal of Neuroscience, 20, 2441-2446.

Davies, R. R., Xuereb, J. H., \& Hodges, J. R. (2002). The human perirhinal cortex in semantic memory: An in vivo and postmortem volumetric magnetic resonance imaging study in semantic dementia, Alzheimer's disease and matched controls. Neuropathology and Applied Neurobiology, 28, 167-168.

Devlin, J. T., \& Price, C. J. (2007). Perirhinal contributions to human visual perception. Current Biology, 17, 1484-1488.

Diana, R. A., Yonelinas, A. P., \& Ranganath, C. (2007). Imaging recollection and familiarity in the medial temporal lobe: A three-component model. Trends in Cognitive Sciences, 11, 379-386.

Diana, R. A., Yonelinas, A. P., \& Ranganath, C. (2008). Highresolution multi-voxel pattern analysis of category selectivity in the medial temporal lobes. Hippocampus, 18, 536-541.

Eichenbaum, H., \& Cohen, N. J. (2001). From conditioning to conscious recollection: Memory systems of the brain. New York: Oxford University Press.

Friston, K. J., Fletcher, P., Josephs, O., Holmes, A., Rugg, M. D., \& Turner, R. (1998). Event-related fMRI: Characterizing differential responses. Neuroimage, 7, 30-40.

Friston, K. J., Penny, W., Phillips, C., Kiebel, S., Hinton, G., \& Ashburner, J. (2002). Classical and Bayesian inference in neuroimaging: Theory. Neuroimage, 16, 465-483.

Galton, C. J., Patterson, K., Graham, K., Lambon-Ralph, M. A., Williams, G., Antoun, N., et al. (2001). Differing patterns of temporal atrophy in Alzheimer's disease and semantic dementia. Neurology, 57, 216-225.

Gauthier, I., \& Tarr, M. J. (1997). Becoming a "Greeble" expert: Exploring mechanisms for face recognition. Vision Research, 37, 1673-1682.

Gorno-Tempini, M. L., Pradelli, S., Serafini, M., Pagnoni, G., Baraldi, P., Porro, C., et al. (2001). Explicit and incidental facial expression processing: An fMRI study. Neuroimage, 14, 465-473.

Gorno-Tempini, M. L., Price, C. J., Josephs, O., Vandenberghe, R., Cappa, S. F., Kapur, N., et al. (1998). The neural systems sustaining face and proper-name processing. Brain, 121, 2103-2118.

Grabowski, T. J., Damasio, H., Tranel, D., Ponto, L. L., Hichwa, R. D., \& Damasio, A. R. (2001). A role for left temporal pole in the retrieval of words for unique entities. Human Brain Mapping, 13, 199-212.

Graham, K. S., Barense, M. D., \& Lee, A. C. (2010). Going beyond LTM in the MTL: A synthesis of neuropsychological and neuroimaging findings on the role of the medial 
temporal lobe in memory and perception. Neuropsychologia, 48, 831-853.

Henson, R. N. A., \& Penny, W. D. (2003). ANOVAs and SPM. Technical Report, Wellcome Department of Imaging Neuroscience.

Holdstock, J. S., Hocking, J., Notley, P., Devlin, J. T., \& Price, C. J. (2009). Integrating visual and tactile information in the perirhinal cortex. Cerebral Cortex, 19, 2993-3000.

Kensinger, E. A., \& Schacter, D. L. (2006). Amygdala activity is associated with the successful encoding of item, but not source, information for positive and negative stimuli. Journal of Neuroscience, 26, 2564-2570.

Kirwan, C. B., \& Stark, C. E. (2004). Medial temporal lobe activation during encoding and retrieval of novel face-name pairs. Hippocampus, 14, 919-930.

Kumaran, D., \& Maguire, E. A. (2006a). The dynamics of hippocampal activation during encoding of overlapping sequences. Neuron, 49, 617-629.

Kumaran, D., \& Maguire, E. A. (2006b). An unexpected sequence of events: Mismatch detection in the human hippocampus. PLoS Biology, 4, e424.

Kumaran, D., \& Maguire, E. A. (2007). Match mismatch processes underlie human hippocampal responses to associative novelty. Journal of Neuroscience, 27, 8517-8524.

Lambon Ralph, M. A., Lowe, C., \& Rogers, T. T. (2007). Neural basis of category-specific semantic deficits for living things: Evidence from semantic dementia, HSVE and a neural network model. Brain, 130, 1127-1137.

Lee, A. C., Barense, M. D., \& Graham, K. S. (2005). The contribution of the human medial temporal lobe to perception: Bridging the gap between animal and human studies. Quarterly Journal of Experimental Psychology B, $58,300-325$

Lee, A. C., Buckley, M. J., Gaffan, D., Emery, T., Hodges, J. R., \& Graham, K. S. (2006). Differentiating the roles of the hippocampus and perirhinal cortex in processes beyond long-term declarative memory: A double dissociation in dementia. Journal of Neuroscience, 26, 5198-5203.

Lee, A. C., Buckley, M. J., Pegman, S. J., Spiers, H., Scahill, V. L., Gaffan, D., et al. (2005). Specialization in the medial temporal lobe for processing of objects and scenes. Hippocampus, 15, 782-797.

Lee, A. C., Bussey, T. J., Murray, E. A., Saksida, L. M., Epstein, R. A., Kapur, N., et al. (2005). Perceptual deficits in amnesia: Challenging the medial temporal lobe "mnemonic" view. Neuropsychologia, 43, 1-11.

Lee, A. C., \& Rudebeck, S. R. (2010). Human medial temporal lobe damage can disrupt the perception of single objects Journal of Neuroscience, 30, 6588-6594.

Lee, A. C., Scahill, V. L., \& Graham, K. S. (2008). Activating the medial temporal lobe during oddity judgment for faces and scenes. Cerebral Cortex, 18, 683-696.

Lemieux, L., Salek-Haddadi, A., Lund, T. E., Laufs, H., \& Carmichael, D. (2007). Modelling large motion events in fMRI studies of patients with epilepsy. Magnetic Resonance Imaging, 25, 894-901.

Leveroni, C. L., Seidenberg, M., Mayer, A. R., Mead, L. A., Binder, J. R., \& Rao, S. M. (2000). Neural systems underlying the recognition of familiar and newly learned faces. Journal of Neuroscience, 20, 878-886.

Levy, D. A., Bayley, P. J., \& Squire, L. R. (2004). The anatomy of semantic knowledge: Medial vs. lateral temporal lobe. Proceedings of the National Academy of Sciences, U.S.A., 101, 6710-6715.

Litman, L., Awipi, T., \& Davachi, L. (2009). Category-specificity in the human medial temporal lobe cortex. Hippocampus, 19, 308-319.
MacKay, D. G., \& James, L. E. (2009). Visual cognition in amnesic H.M.: Selective deficits on the What's-Wrong-Here and Hidden-Figure tasks. Journal of Clinical and Experimental Neuropsychology, 31, 769-789.

MacKay, D. G., James, L. E., \& Hadley, C. B. (2008). Amnesic H.M.'s performance on the language competence test: Parallel deficits in memory and sentence production. Journal of Clinical and Experimental Neuropsychology, 30, 280-300.

McClelland, J. L., \& Rogers, T. T. (2003). The parallel distributed processing approach to semantic cognition. Nature Reviews Neuroscience, 4, 310-322.

Moses, S. N., Ostreicher, M. L., Rosenbaum, R. S., \& Ryan, J. D. (2008). Successful transverse patterning in amnesia using semantic knowledge. Hippocampus, 18, 121-124.

Mummery, C. J., Patterson, K., Price, C. J., Ashburner, J., Frackowiak, R. S., \& Hodges, J. R. (2000). A voxel-based morphometry study of semantic dementia: Relationship between temporal lobe atrophy and semantic memory. Annals of Neurology, 47, 36-45.

Mundy, M. E., Honey, R. C., Downing, P. E., Wise, R. G., Graham, K. S., \& Dwyer, D. M. (2009). Material-independent and material-specific activation in functional MRI after perceptual learning. NeuroReport, 20, 1397-1401.

Murray, E. A., \& Bussey, T. J. (1999). Perceptual-mnemonic functions of the perirhinal cortex. Trends in Cognitive Sciences, 3, 142-151.

Murray, E. A., Bussey, T. J., \& Saksida, L. M. (2007). Visual perception and memory: A new view of medial temporal lobe function in primates and rodents. Annual Review of Neuroscience, 30, 99-122.

Nakamura, K., Kawashima, R., Sato, N., Nakamura, A., Sugiura, M., Kato, T., et al. (2000). Functional delineation of the human occipito-temporal areas related to face and scene processing. A PET study. Brain, 123, 1903-1912.

Olson, I. R., Plotzker, A., \& Ezzyat, Y. (2007). The enigmatic temporal pole: A review of findings on social and emotional processing. Brain, 130, 1718-1731.

O’Neil, E. B., Cate, A. D., \& Kohler, S. (2009). Perirhinal cortex contributes to accuracy in recognition memory and perceptual discriminations. Journal of Neuroscience, 29, 8329-8334.

Otten, L. J., Henson, R. N. A., \& Rugg, M. D. (2001). Depth of processing effects on neural correlates of memory encoding: Relationship between findings from across- and within-task comparisons. Brain, 124, 399-412.

Patterson, K., Nestor, P. J., \& Rogers, T. T. (2007). Where do you know what you know? The representation of semantic knowledge in the human brain. Nature Reviews Neuroscience, 8, 976-987.

Preston, A. R., Bornstein, A. M., Hutchinson, J. B., Gaare, M. E., Glover, G. H., \& Wagner, A. D. (2010). High-resolution fMRI of content-sensitive subsequent memory responses in human medial temporal lobe. Journal of Cognitive Neuroscience, 22, 156-173.

Ranganath, C., Yonelinas, A. P., Cohen, M. X., Dy, C. J., Tom, S. M., \& D'Esposito, M. (2004). Dissociable correlates of recollection and familiarity within the medial temporal lobes. Neuropsychologia, 42, 2-13.

Rogers, T. T., Lambon Ralph, M. A., Garrard, P., Bozeat, S., McClelland, J. L., Hodges, J. R., et al. (2004). Structure and deterioration of semantic memory: A neuropsychological and computational investigation. Psychological Review, 111, 205-235.

Rosen, H. J., Gorno-Tempini, M. L., Goldman, W. P., Perry, R. J., Schuff, N., Weiner, M., et al. (2002). Patterns of brain atrophy in frontotemporal dementia and semantic dementia. Neurology, 58, 198-208. 
Ross, L. A., \& Olson, I. R. (2010). Social cognition and the anterior temporal lobes. Neuroimage, 49, 3452-3462.

Schwarzbauer, C., Mildner, T., Heinke, W., Brett, M., \& Deichmann, R. (2010). Dual echo EPI-The method of choice for fMRI in the presence of magnetic field inhomogeneities? Neuroimage, 49, 316-326.

Sergent, J., Ohta, S., \& MacDonald, B. (1992). Functional neuroanatomy of face and object processing. A positron emission tomography study. Brain, 115, 15-36.

Simmons, W. K., Reddish, M., Bellgowan, P. S., \& Martin, A. (2010). The selectivity and functional connectivity of the anterior temporal lobes. Cerebral Cortex, 20, 813-825.

Squire, L. R., Stark, C. E., \& Clark, R. E. (2004). The medial temporal lobe. Annual Review of Neuroscience, 27, 279-306.

Squire, L. R., \& Zola-Morgan, S. (1991). The medial temporal lobe memory system. Science, 253, 1380-1386.

Suzuki, W. A., \& Amaral, D. G. (1994). Perirhinal and parahippocampal cortices of the macaque monkey: Cortical afferents. Journal of Comparative Neurology, 350, 497-533.

Taylor, K. I., Stamatakis, E. A., \& Tyler, L. K. (2009). Crossmodal integration of object features: Voxel-based correlations in brain-damaged patients. Brain, 132, 671-683.

Taylor, K. J., Henson, R. N. A., \& Graham, K. S. (2007). Recognition memory for faces and scenes in amnesia: Dissociable roles of medial temporal lobe structures. Neuropsychologia, 45, 2428-2438.

Trinkler, I., King, J. A., Doeller, C. F., Rugg, M. D., \& Burgess, N. (2009). Neural bases of autobiographical support for episodic recollection of faces. Hippocampus, 19, 718-730.
Tzourio-Mazoyer, N., Landeau, B., Papathanassiou, D., Crivello, F., Etard, O., Delcroix, N., et al. (2002). Automated anatomical labeling of activations in SPM using a macroscopic anatomical parcellation of the MNI MRI single-subject brain. Neuroimage, 15, 273-289.

Uncapher, M. R., \& Rugg, M. D. (2005). Encoding and the durability of episodic memory: A functional magnetic resonance imaging study. Journal of Neuroscience, 25, 7260-7267.

Visser, M., Jefferies, E., \& Lambon Ralph, M. A. (2010). Semantic processing in the anterior temporal lobes: A meta-analysis of the functional neuroimaging literature. Journal of Cognitive Neuroscience, 22, 1083-1094.

Wagner, A. D., Schacter, D. L., Rotte, M., Koutstaal, W., Maril, A., Dale, A. M., et al. (1998). Building memories: Remembering and forgetting of verbal experiences as predicted by brain activity. Science, 281, 1188-1191.

Wang, W. C., Lazzara, M. M., Ranganath, C., Knight, R. T., \& Yonelinas, A. P. (2010). The medial temporal lobe supports conceptual implicit memory. Neuron, 68, 835-842.

Worsley, K. J., Marrett, S., Neelin, P., Vandal, A. C., Friston, K. J., \& Evans, A. C. (1995). A unified approach for determining significant signals in images of cerebral activation. Human Brain Mapping, 4, 58-73.

Zahn, R., Moll, J., Iyengar, V., Huey, E. D., Tierney, M., Krueger, F., et al. (2009). Social conceptual impairments in frontotemporal lobar degeneration with right anterior temporal hypometabolism. Brain, 132, 604-616.

Zahn, R., Moll, J., Krueger, F., Huey, E. D., Garrido, G., \& Grafman, J. (2007). Social concepts are represented in the superior anterior temporal cortex. Proceedings of the National Academy of Sciences, U.S.A., 104, 6430-6435. 